\title{
Psychological Distress Among Healthcare Providers During the COVID-19 Pandemic in Gaza Strip: A Cross-sectional Study
}

\author{
Osama Jabr Emad 1, 2, *, Abdalkarim Said Radwan², Hassan Mohammed Abu Rhama ${ }^{3}$, \\ Mohammed Jaser Afana ${ }^{3}$ \\ ${ }^{1}$ Research Department, Ministry of Health, General Mental Health Directorate, Gaza, Palestine \\ ${ }^{2}$ Faculty of Nursing, Islamic University, Gaza, Palestine \\ ${ }^{3}$ General Mental Health Directorate, Gaza, Palestine \\ Email address: \\ Oemad207567@gmail.com (O. J. Emad), arodwan@iugaza.edu.ps (A. S. Radwan), scbu2010@gmail.com (H. M. Abu Rhama), \\ moafana9@gmail.com (M. J. Afana) \\ ${ }^{*}$ Corresponding author
}

\section{To cite this article:}

Osama Jabr Emad, Abdalkarim Said Radwan, Hassan Mohammed Abu Rhama, Mohammed Jaser Afana. Psychological Distress Among Healthcare Providers During the COVID-19 Pandemic in Gaza Strip: A Cross-sectional Study. International Journal of Psychological and Brain Sciences. Vol. 6, No. 1, 2021, pp. 13-16. doi: 10.11648/j.ijpbs.20210601.13

Received: February 19, 2021; Accepted: March 11, 2021; Published: March 26, 2021

\begin{abstract}
Background: Healthcare providers are engaged in providing patient care and may expose to COVID-19 during their work, Understanding the psychological impact of the COVID-19 among health care providers is important in facing the pandemic. Objective: We examined the psychological distress, depression, anxiety, and stress among healthcare providers during the COVID-19 Pandemic in Gaza Strip. Methods: The descriptive design was used, 231 health care providers for corona patients were selected purposively, Depression, Anxiety, and Stress Scales (DASS-21) In addition to information on demographic characteristics was used in this study. Results: The mean weight for depression domain $68 \%$ and the mean weight for anxiety $69 \%$, on the other hand, the mean weight for stress $67 \%$, the study show that Mean Rank and the p-value equal 0.693, which is greater than 0.05 , which means there are no significant differences between psychological distresses due to gender among healthcare providers during the Covid-19 pandemic, the study found that the p-value $<0.05$, which means there are significant differences between psychological distresses due to years of experience in favor of more than 12 years and type of job in favor of Nonmedical Health Care Personnel. Conclusion: Our study highlights that nonmedical health care personnel are at the highest risk for psychological distress during the COVID-19 pandemic.
\end{abstract}

Keywords: COVID-19, Psychological Distress, Health Providers

\section{Introduction}

The coronavirus COVID-19 pandemic has become a global health crisis that significantly affects the international health system across the world. Since the start of the Corona pandemic globally, all travelers coming through Rafah and Beit Hanon (Erez) crossings had undergone a compulsory quarantine at one of the Ministry of Health (MOH) designated facilities, isolation hospitals, and health quarantine centers. People stay for 21 days in quarantine centers, health services are provided, and laboratory tests are carried out by the medical staff present with them around the clock, according to the $\mathrm{MOH}$, the number of service providers in the quarantine centers reached 389 employees, $20 \%$ are a medical team, On 26 August 2020, MOH in Gaza confirmed the first COVID-19 spread through the community in the Gaza strip. When preparing this study, the total number of confirmed cases is 53440, of which 2949 are still active cases, 49955 cases fully recovered and were discharged, while the number of deaths reached 536 deaths [1].

Healthcare providers are engaged in providing patient care and may expose to COVID-19 during their work. Moreover, they are not only at risk for physical challenges but also mental 
burdens, particularly psychological distress [2]. Healthcare workers are facing unprecedented amounts of COVID-19related psychological stress across professional and personal domains [3]. The adverse psychological impact includes acute stress disorder, insomnia, post-traumatic symptoms, and depression [4]. The unusual circumstances in which the medical team works and their presence in the same place for 21 days with people infected with or suspected of being infected with the virus in addition to their anxiety for themselves and their families, these factors considers sources of anxiety, stress, and depression for health care providers. COVID-19 has resulted in high levels of uncertainty, anxiety, social isolation, and further inequity in health care for Palestinians in Gaza [5]. World Health Organization (WHO) issued a COVID-19 guideline on mental health and psychological distress to support people's mental and psychological wellbeing during this outbreak [6]. Moreover, we mean here by psychological distress that it includes the emotional state of depression, anxiety, and stress. Anxiety is defined as irrational and excessive fear, apprehensive, and tense feelings difficulty managing daily tasks, and/or distress related to these tasks [7]. People with experience a range of symptoms including persistent depressed mood or loss of interest and pleasure for at least 2 weeks suffer from depression [8]. Hans Selye defines stress as the "perception of threat, with resulting anxiety discomfort, emotional tension, and difficulty in adjustment." [9]. The present study will provide recommendations to deal with psychological problems during the COVID-19 pandemic and improve the mental health status among healthcare providers. This study aims to assess the level of the emotional state of depression, anxiety, and stress among health care providers during the COVID-19 in Gaza Strip.

\section{Methodology}

\subsection{Study Design}

The design of this study cross sectional design.

\subsection{The Study Population}

Health care providers working with covide 19 infected patients (physicians, Nurses, Nonmedical Health Care Personnel)

\subsection{Sample}

The sample consists of 231 healthcare providers for Covid 19 patients who work at the Turkish Friendship Hospital and the European Gaza Hospital. The type of sample is purposive sample (Non-probability).

\subsection{Instruments}

Validated Depression, Anxiety, and Stress Scales (DASS-21) In addition to information on demographic characteristics [10].

\subsection{Procedure}

From 15 August to 20 October 2020, health care workers from 2 major hospitals in Gaza who were caring for patients with COVID-19 were invited to participate with a selfadministered questionnaire (online).

\section{Results}

\subsection{Level of Depression, Anxiety, and Stress Among Healthcare Providers During the COVID-19 Pandemic in Gaza Strip}

Table 1. Description of respondents' psychological distress $(n=231)$.

\begin{tabular}{lllllll}
\hline Weight mean \% & Mean & Very sever & Severe & Moderate & Mild & Normal \\
\hline $68 \%$ & 2.0433 & 162 & 8 & 20 & 22 & 19 \\
$69 \%$ & 2.0816 & 174 & 11 & 27 & 10 & 9 \\
$67 \%$ & 2.0198 & 129 & 36 & 5 & 11 & Anxiety \\
\hline
\end{tabular}

Table 1 shows that the level of depression, anxiety, and stress among health care providers during the COVID-19 Pandemic. In the depression domain, 19 participants are normal degrees, 22 from participants were mild degrees and 20 were moderate while 8 participant's severe degrees and 162 were very severed. The level of depression on the whole depression scale equals $68 \%$. On other hand, in the anxiety domain, 9 participants were normal degree, 10 participants were mild degree and 27 were moderate, 11 were severe degree, while 174 participants were a very severe degree. The level of anxiety on the whole anxiety scale equals $69 \%$. In contrast, in the stress domain, 50 participants were normal degree, 11 participants were mild degree and 5 moderate degrees, 36 participants severe while 129 were very severed. The level of stress on the whole stress scale equals $67 \%$.

\subsection{The Relationship Between Depression, Anxiety, and Stress Level Due to Gender Among Healthcare Providers During the COVID-19 Pandemic in Gaza Strip}

Table 2. Mann-Whitney test to view up the relationship between psychological distresses due to gender among healthcare providers during the Covid-19 pandemic.

\begin{tabular}{llllll}
\hline items & Gender & N & Mean Rank & Sum of Ranks & Z \\
\hline \multirow{3}{*}{ Psychological distress } & Male & 150 & 114.73 & 17209.00 & \\
& Female & 81 & 118.36 & 9587.00 & -0.395 \\
& Total & 231 & & & \\
\hline
\end{tabular}

$* \mathrm{P}<0.05$ 
Table 2 shows that Mean Rank and the p-value equal 0.693, which is greater than 0.05 , which means there are no significant differences between psychological distresses due to gender among healthcare providers during the Covid-19 pandemic

\subsection{The Relationship Between Depression, Anxiety, and Stress Due to Years of Experiences Among Health Providers During the COVID-19 Pandemic in Gaza Strip}

Table 3. Kruskal-Wallis test to view up the relationship between psychological distresses due to years of experiences among healthcare providers during the Covid-19 pandemic.

\begin{tabular}{lllll}
\hline Items & experience & N & Mean Rank & df \\
\hline \\
Psychological & below 5 years & 84 & 121.76 & \\
distress & 5-12 years & 75 & 99.40 & 2 \\
& up 12 years & 72 & 126.57 & $0.02 *$ \\
\hline
\end{tabular}

$\mathrm{P}<0.05$

Table 3 shows the relationship between psychological distresses due to years of experiences by using the KruskalWallis test. The p- values are less than 0.05 in psychological distress scores, thus there is a significant relationship between psychological distresses due to years of experiences in favor of more than 12 years' experience among healthcare providers during the COVID-19 pandemic.

\subsection{The Relationship Between Psychological Distresses and Type of Occupation Among Health Providers During the COVID-19 Pandemic in Gaza Strip}

Table 4. Kruskal-Wallis test to view up the relationship between psychological distresses levels due to type of occupation of healthcare providers during the Covid-19 pandemic.

\begin{tabular}{lllll}
\hline Item & Professions & N & Mean Rank & df \\
\hline & Nurse & 152 & 105.41 & \\
Psychological distress & Doctor & 27 & 127.87 & 2 \\
& Nonmedical Health Care Personnel & 52 & 140.78 & \\
& Total & 231 & & \\
\hline
\end{tabular}

$* \mathrm{P}<0.05$

Table 4 shows that the relationship between psychological distress due to occupation by using $\mathrm{K}$ independent variables test. The p- values are less than 0.05 , which means there is a significant difference between psychological distresses to occupation in favor of Nonmedical Health Care Personnel as (administrators, maintenance workers).

\section{Discussion}

Overall mean DASS-21 among health care workers were higher than those in the published previous studies as $[2,11$, 12] which showed that an almost 2 times lower level of DASS-21, than those in our study. This could be attributed to in addition to the risks of infection from Corona and work stress, health care providers suffer from difficult economic, social, and political conditions such as low salary, lack of human staff, sieges and wars, all these factors and others, in addition to the Corona pandemic, have contributed to the increase of severe symptoms in levels of depression, anxiety and stress among health care providers. Our study agree with study $46.5 \%$ were mild to moderately distresse and $6.7 \%$ were severely distressed due to the current COVID-19 pandemic [13]. The prevalence of mild distress was reported to be lower among health workers from China (36.5\%) [14]and in Saudi Arabia (33.7\%) [15] as compared to the findings of our study.
The present study show that there are no significant differences between psychological distresses due to gender among healthcare providers during the Covid-19 pandemic, this result disagrees with another study[11] which show that the largest distress burden falls on females relative to males.

On other hand, the study found that there are significant differences between psychological distresses due to years of experience in favor of more than 12 years and type of job in favor of Nonmedical Health Care Personnel. This result agree with other study [2, 12]. Reasons for this may include increase in working years with the continuing difficult working conditions and sources of stress, in addition to the Corona pandemic, leads to the person being exposed to severe levels of anxiety, depression and stress, On the other hand, Nonmedical Health Care Personnel are less information on the corona, less intensive training on personal protective equipment and infection control measures. In addition, Nonmedical Health Care Personnel are not included in the psychological support that targets medical teams only.

As the pandemic continues, psychosocial intervention program are needed to support health care providers, our study identified vulnerable groups who are more at risk to psychological distress. Self-care program and educational intervention should target nonmedical health care personnel to increase their awareness about mental health.

The study suggests activating a face-to-face psychological 
counseling service or by calling to support medical and nonmedical health care personnel in facing the pandemic, teaching them positive coping methods, and providing useful psychological advice and guidance to them to reduce the level of depression, anxiety and stress.

\section{Conclusion}

Our study highlights that nonmedical health care personnel are at the highest risk for psychological distress during the COVID-19 pandemic. This study emphasizes the need to pay attention not only to people but also to health care providers during the COVID-19 pandemic, We hope that this descriptive study will contribute to understanding how the COVID-19 pandemic affect mental health among health care providers Early psychological interventions like helping the helpers program targeting this vulnerable group may be beneficial and important to provide counseling and psychological support to them when needed so that they are able to cope with stress and life problems.

\section{Disclosure Statement}

No financial interest or any conflict of interest exists.

\section{Acknowledgements}

Special thanks to our colleagues in the Mental Health General Directorate $\mathrm{MOH}$ - who cooperated with us to complete this study.

\section{References}

[1] Ministry Of Health (2021) daily report for COVID19 Virus, unit of information system, Gaza, Palestine.

[2] Lai, J. Ma, S. Wang, Y. Cai, Z. Hu, J. Wei, N. Tan, H. (2020) Factors associated with mental health outcomes among health care workers exposed to coronavirus disease 2019. JAMA network open, 3 (3), e203976-e203976.

[3] Shanafelt, T. Ripp, J. Trockel, M. (2020). Understanding and addressing sources of anxiety among health care professionals during the COVID-19 pandemic. Jama, 323 (21), 2133-2134.

[4] Brooks, S. K. Webster, R. K. Smith, L. E. Woodland, L. Wessely, S. Greenberg, N. Rubin, G. J. (2020) the psychological impact of quarantine and how to reduce it: rapid review of the evidence. The Lancet.

[5] Alser, O. AlWaheidi, S. Elessi, K. Meghari, H. (2020). COVID-19 in Gaza: a pandemic spreading in a place already under protracted lockdown. Eastern Mediterranean Health Journal, 26 (7), 762-763.

[6] World Health Organization (2020) Mental health and psychosocial considerations during the COVID-19 outbreak, 18 March 2020 (No. WHO/2019-nCoV/Mental Health/2020.1). World Health Organization.

[7] Rector, N. (2011) Anxiety disorders, an information guide, Centre for Addiction and Mental Health. A pan American Health organization, world health organization.

[8] World Health Organization (2016) mhGAP intervention guide for mental, neurological and substance use disorders in nonspecialized health settings: mental health Gap Action Programme (mhGAP) - version 2.0. ISBN 9789241549790.

[9] Fink, G. (2016) Stress concepts and cognition, emotion, and behavior. (1 ed.). Amsterdam: Academic Press.

[10] Lovibond SH, Lovibond PF (1995) Manual for the Depression Anxiety Stress Scales. 2d ed. Sydney, Psychology Foundation of Australia.

[11] Al-Hanawi, M. K. Mwale, M. L. Alshareef, N. Qattan, A. M. Angawi, K. Almubark, R. Alsharqi, O. (2020). Psychological Distress amongst Health Workers and the General Public during the COVID-19 Pandemic in Saudi Arabia. Risk Management and Healthcare Policy, 13, 733.

[12] Tan, B. Y. Chew, N. W. Lee, G. K. Jing, M. Goh, Y. Yeo, L. L. Shanmugam, G. N. (2020). Psychological impact of the COVID-19 pandemic on health care workers in Singapore. Annals of Internal Medicine.

[13] Kafle K, Shrestha DB, Baniya A, Lamichhane S, Shahi M, Gurung B, et al. (2021) Psychological distress among health service providers during COVID-19 pandemicin Nepal. $\begin{array}{llll}\text { PLoSONE } & 16 & \text { (2): } & \text { e0246784. }\end{array}$ https://doi.org/10.1371/journal.pone.0246784.

[14] Bao Y, SunY, Meng S, ShiJ, LuL. 2019- nCoV epidemic: address mental health care to empower society. Lancet. 2020; 395: e37-e38. https://doi.org/10.1016/S0140-6736(20)303093PMID:3204398.

[15] Shrestha B, Thapa B B, Katuwal N, Shrestha B, Pant C, Basnet B, et al. Psychological distress in Nepalese residents during COVID-19 pandemic: a community-level survey Psychiatry. 2020; 20: 491. https://doi.org/10.1186/s12888020-02904-6 PMID:33023563 